\title{
A one-step cloning method for the construction of somatic cell gene targeting vectors: application to production of human knockout cell lines
}

\author{
Yi Liư ${ }^{\dagger}$, Shangze Li ${ }^{\dagger}$, Huihui Zhang, Zurong Wan, Xiaodong Zhang and Runlei Du*
}

\begin{abstract}
Background: Gene targeting is a powerful method that can be used for examining the functions of genes. Traditionally, the construction of knockout $(\mathrm{KO})$ vectors requires an amplification step to obtain two homologous, large fragments of genomic DNA. Restriction enzymes that cut at unique recognitions sites and numerous cloning steps are then carried out; this is often a time-consuming and frustrating process.

Results: We have developed a one-step cloning method for the insertion of two arms into a KO vector using exonuclease III. We modified an adeno-associated virus KO shuttle vector (pTK-LoxP-NEO-AAV) to yield pAAV-LIC, which contained two cassettes at the two multiple-cloning sites. The vector was digested with EcoRV to give two fragments. The two homologous arms, which had an overlap of 16 bases with the ends of the vector fragments, were amplified by polymerase chain reaction. After purification, the four fragments were mixed and treated with exonuclease III, then transformed into Escherichia coli to obtain the desired clones. Using this method, we constructed SirT1 and HDAC2 KO vectors, which were used to establish SirT1 KO cells from the colorectal cancer cell line (HCT116) and HDAC2 KO cells from the colorectal cancer cell line (DLD1).
\end{abstract}

Conclusions: Our method is a fast, simple, and efficient technique for cloning, and has great potential for high-throughput construction of $\mathrm{KO}$ vectors.

\section{Background}

Gene targeting is a powerful method for the production of genetically modified cell lines or animals, allowing for the various functions of genes to be studied. In mice, several thousand genes have been disrupted using homologous recombination. However, when these methods have been applied to human somatic cells they have generally been ineffective because of very low targeting efficiencies [1]. Although RNA interference can reduce the expression of a gene, interpretation of such experiments can be unreliable because of non-specific targeting or incomplete inactivation of genes. Therefore, we believe it is essential to improve the efficiency of knockout (KO) approaches in human somatic cells.

Recombinant adeno-associated viruses (rAAVs), such as human parvovirus, possess a single-stranded DNA genome of around $4.7 \mathrm{~kb}$. Hirata [2] and Porteus [3]

\footnotetext{
* Correspondence: runleidu@whu.edu.cn

${ }^{\dagger}$ Equal contributors

College of Life Sciences, Wuhan University, Wuhan, Hubei, PR China
}

found that rAAVs can be used to target genes in human cell lines. Use of these rAAVs resulted in higher targeting frequencies than those obtained with conventional plasmid vectors $[4,5]$. The wild-type human parvovirus genome contains two open reading frames (ORFs), designated rep and cap, flanked by two inverted terminal repeats. The rep ORF encodes proteins involved in viral replication, and the cap ORF encodes proteins necessary for viral packaging. In $\mathrm{rAAV}$-mediated $\mathrm{KO}$ vectors these ORFs are deleted and replaced with a neomycin resistant gene flanked by two homology arms. The stages involved in carrying out rAAV-mediated gene KO include: (i) design and construction of the AAV KO vector; (ii) collecting an infectious rAAV stock; (iii) infecting the appropriate cell line; (iv) screening for homologous recombinants; and (v) iteratively targeting the multiple alleles. It takes at least three months just to target the first allele [6].

Apart from the low efficiency of homologous recombination, a rate-limiting step in gene targeting of human 
somatic cell lines is assembly of the gene targeting construct. Traditionally, this requires an amplification step to obtain two large homologous fragments of genomic DNA, followed by restriction endonuclease digestion, and then numerous cloning steps. It is an extremely time-consuming process and limited by the available unique restriction enzyme sites in the vector and in the two amplified homologous fragments. Phage-based Escherichia coli homologous recombination systems [7-9] have been developed that now make it possible to subclone or modify DNA cloned into plasmids, bacterial artificial chromosomes (BACs), or P1-derived artificial chromosomes (PACs) without the need for restriction enzymes or DNA ligases. However, these recombination systems require long homology arms and usually one can only insert one fragment at the time.

Traditional DNA cloning suffers from several limitations, including poor ligation efficiency, along with a dependence on the availability of unique restriction sites in both the insert and vector. For this reason, many methods regarding directional subcloning have been developed $[10,11]$. These methods include the use of uracil DNA N-glycosylase [12], T4 DNA polymerase [13], enzymatic assembly [14-16] or exonuclease III (ExoIII) [17] to generate long compatible cohesive ends between the DNA insert and cloning vector. It has also been found that by generating longer cohesive ends, the annealed DNA complex becomes more stable and the ligation reaction can be omitted. This technique has come to be known as ligation-independent cloning (LIC), and has been demonstrated to have a high efficiency. Developing more LIC methods will give the researchers more chioce according to the different situations.

To increase cloning efficiency and ligation of four fragments simultaneously, we developed a one-step LIC method for construction of $\mathrm{KO}$ vectors. Using this method, it becomes easy to construct $\mathrm{KO}$ vectors in two days. In this paper, we have outlined our strategy for modifying pTK-LoxP-NEO-AAV, and demonstrated successful construction of a SirT1 and HDAC2 KO vector. Once we had made the vectors, we were able to obtain SirT1 KO cells from the colorectal cancer cell line HCT116 and HDAC2 KO cells from the colorectal cancer cell line DLD1.

\section{Results and discussion}

\section{Construction and characterization of a pAAV-LIC vector}

Assembly of gene-targeting constructs is a rate-limiting step when targeting genes in human somatic cell lines. Other limitations include multi-step ligations and the availability of unique restriction sites in the insert and vector. To overcome many of these limitations we modified an AAV shuttle vector into a one-step LIC vector. This was achieved by introducing two fixed cassettes into the multiple-cloning site (MCS) of the shuttle vector (Figure 1). The two cassettes involved three elements: sticky ends complementary to the cloning site in pTK-LoxP-NEO-AAV, allowing for insertion of the cassettes; EcoRV sites in the middle of the cassette, which could be used to cut the LIC vector into two fragments containing blunt ends; and 14- to 18-mer sequences that could produce long sticky ends when ExoIII was used.

First, cassette A was inserted into pTK-LoxP-NEO-AAV using EcoRV and SalI digestion. Because the cassette end did not include the EcoRV palindromic sequence, this process restored the SalI site and destroyed the original EcoRV site. Following confirmation by sequencing, cassette B was inserted into the vector now containing cassette A. This was facilitated by digestion with NheI and SpeI digestion. The modifications to the vector were again confirmed by sequencing, and we designated the designed vector as pAAV-LIC.

\section{Construction of a SirT1 and HDAC2 KO vectors}

We used pAAV-LIC to construct SirT1 and HDAC2 KO vector by one-step LIC (Figure 2). The pAAV-LIC vector was digested with EcoRV, resulting in two fragments (approximately $1 \mathrm{~kb}$ and $3.1 \mathrm{~kb}$ ). These fragments were recovered from agarose gels following electrophoresis and purified using gel extraction kits. The smaller fragment was found to contain the thymidine kinase (TK) promoter, the LoxP sequence and the neomycin ORF. The larger fragment was found to contain the AAV backbone, necessary for plasmid replication in E. coli, and for viral packaging.

The two homologous arms for SirT1 and HDAC2 KO vector construction were obtained by polymerase chain reaction (PCR) and purified using gel extraction kits. The PCR primers used were designed to add additional 14 - to 18-mer sequences overlapping with the ends of digested vector fragments. Polymerases, including Taq polymerase, possess the ability to add dATP at the $3^{\prime}$ ends of PCR products, thereby inhibiting the $3^{\prime}$ exonuclease activity of ExoIII. Therefore we recommend using $p f u$ or PrimeStar polymerases when amplifying homologous arms, as they result in amplicons with blunt ends.

The $3^{\prime}$ exonuclease activity of ExoIII is very high at $37^{\circ} \mathrm{C}$. To generate 16 -mer overhangs at a stable and low rate, it is necessary to optimize reaction temperature and time. According to our experience, to ensure stable cloning, $1 \mathrm{~h}$ at $0^{\circ} \mathrm{C}$ or $30 \mathrm{~min}$ at $4^{\circ} \mathrm{C}$ are both sufficient. Using other conditions proved difficult, with both the number of colonies and the rate of positive transformants acquired highly variable. To produce complementary sticky ends, the purified homologous arms and two vector fragments were mixed and chilled at $0^{\circ} \mathrm{C}$ for 10 min, then treated with $20 \mathrm{U}$ of ExoIII at $0^{\circ} \mathrm{C}$ for $1 \mathrm{~h}$. The 


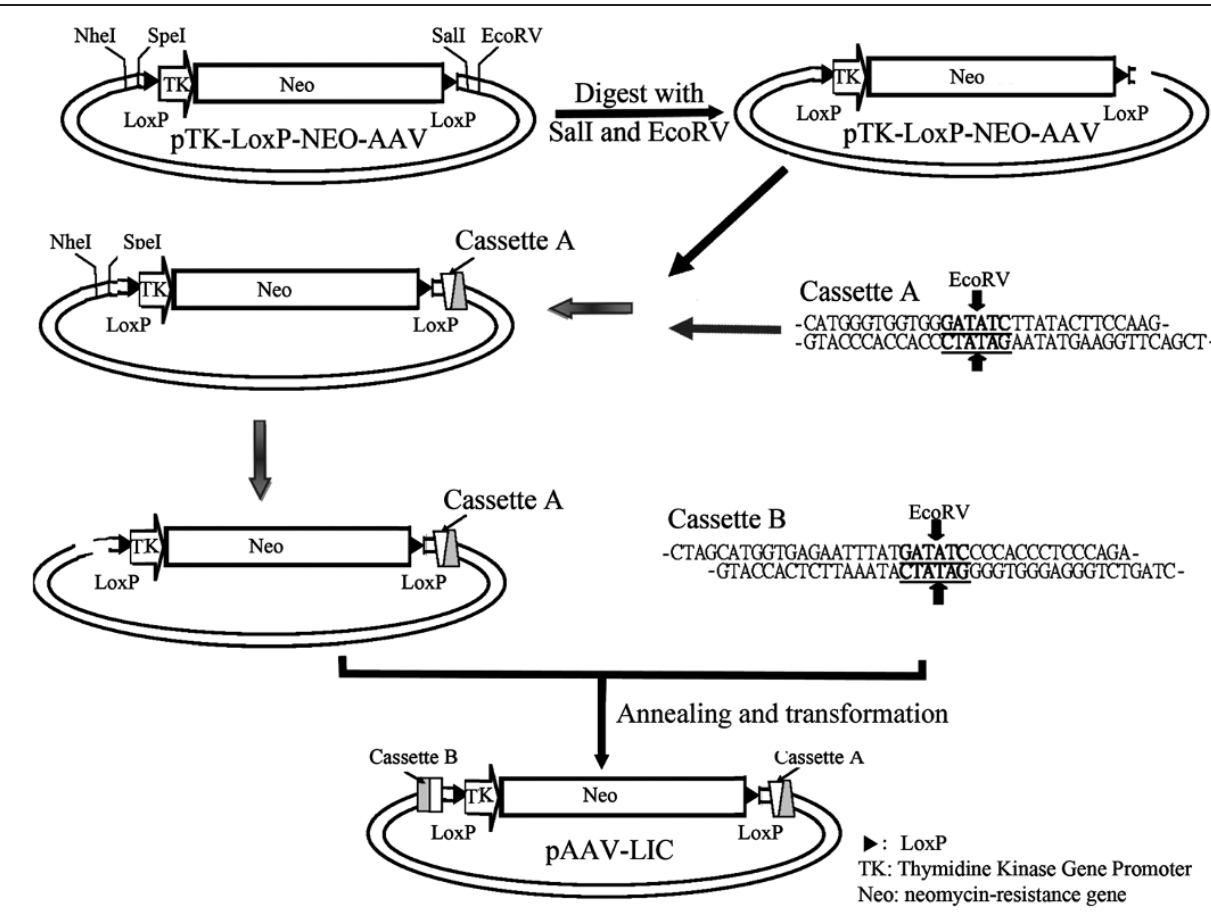

Figure 1 Schematic depicting the modification of pTK-LoxP-NEO-AAV into pAAV-LIC by insertion of two designed LIC adaptors. The two LIC adaptors contain EcoRV sites and an additional 11-15 bp sequence necessary for LIC cloning. The two EcoRV restriction sequences are underlined and an arrow indicates the digestion sites. Left blunt end and right overhang sequences for EcoRV and Sall in cassette $\mathbf{A}$ allow for annealing of the LIC adaptor into the right arm MCS of the PTK-LoxP-NEO-AAV plasmid. Overhang sequences for Nhel and Spel in cassette $\mathbf{B}$ allow for annealing of the LIC adaptor into the left arm MCS.

reaction was terminated using EDTA or by heating to $65^{\circ} \mathrm{C}$. After transformation into $\mathrm{DH} 5 \alpha \mathrm{E}$. coli, enzymatic bacterial DNA repair machinery helped to facilitate ligation endogenously.

Colony PCR and sequencing was used to verify the positive transformants. Screening by PCR showed that 9 of the 12 colonies analyzed were positive transformants (Figure 3a). Sequencing results further confirmed the $\mathrm{PCR}$ results (Figure $3 \mathrm{~b}$ and $\mathrm{c}$ ). The resulting $\mathrm{KO}$ vector was designated as the AAV-SirT1 $\mathrm{KO}$ vector. Also we got the AAV-HDAC2 KO vector.

\section{Use of gene targeting to generate SirT1-null HCT116 cells} and HDAC2-null DLD1 cells

To confirm its ability to be used in the development of $\mathrm{KO}$ cell lines, the targeting AAVs were packaged in 293T cells by transfecting equal amounts of the SirT1 $\mathrm{KO}$ targeting vector targeting vector, pHelper and pRC. Then HCT116 cells were infected with the SirT1 targeting viruses and selected with geneticin. After two weeks, the neomycin-resistant clones were screened for homologous recombination by genomic PCR. We screened 100 clones and obtained 4 positives. To remove the neomycin-resistant gene, two of the correctly targeted clones were infected with adenoviruses expressing Crerecombinase. Using genomic PCR, we screened 24 clones, with 10 of them found to be positives containing an additional $250 \mathrm{bp}$ fragment in which the LoxP site was inserted. Up until this point we had succeeded in obtaining heterozygous $\mathrm{KO}$ clones. To delete both alleles, heterozygous $\mathrm{KO}$ clones were infected with the same targeting virus and the neomycin resistance gene was excised. Finally we obtained homozygous $\mathrm{KO}$ clones and the $\mathrm{KO}$ cell lines were confirmed by western blot (Figure 4a). Use the same protocol, HDAC2 targeting viruses were made and DLD1 cells were infected with the HDAC2 targeting viruses. Also we obtained HDAC2-null DLD1 cells and confirmed by western blot (Figure 4b).

\section{Conclusions}

As outlined above, we successfully constructed the AAVSirT1 and AAV-HDAC2 KO vectors and obtained SirT1 and HDAC2 KO cell lines. Compared with the traditional 'digestion-ligation' vector construction method, our approach is not limited by the availability of restriction sites; therefore, somatic cell gene $\mathrm{KO}$ vectors can be easily constructed within 2-3 days. Additionally, our method does not require the use of any single specific restriction enzyme, and has great potential for the high-throughput construction of gene $\mathrm{KO}$ vectors. However, it also has some limitations, such as the potential 


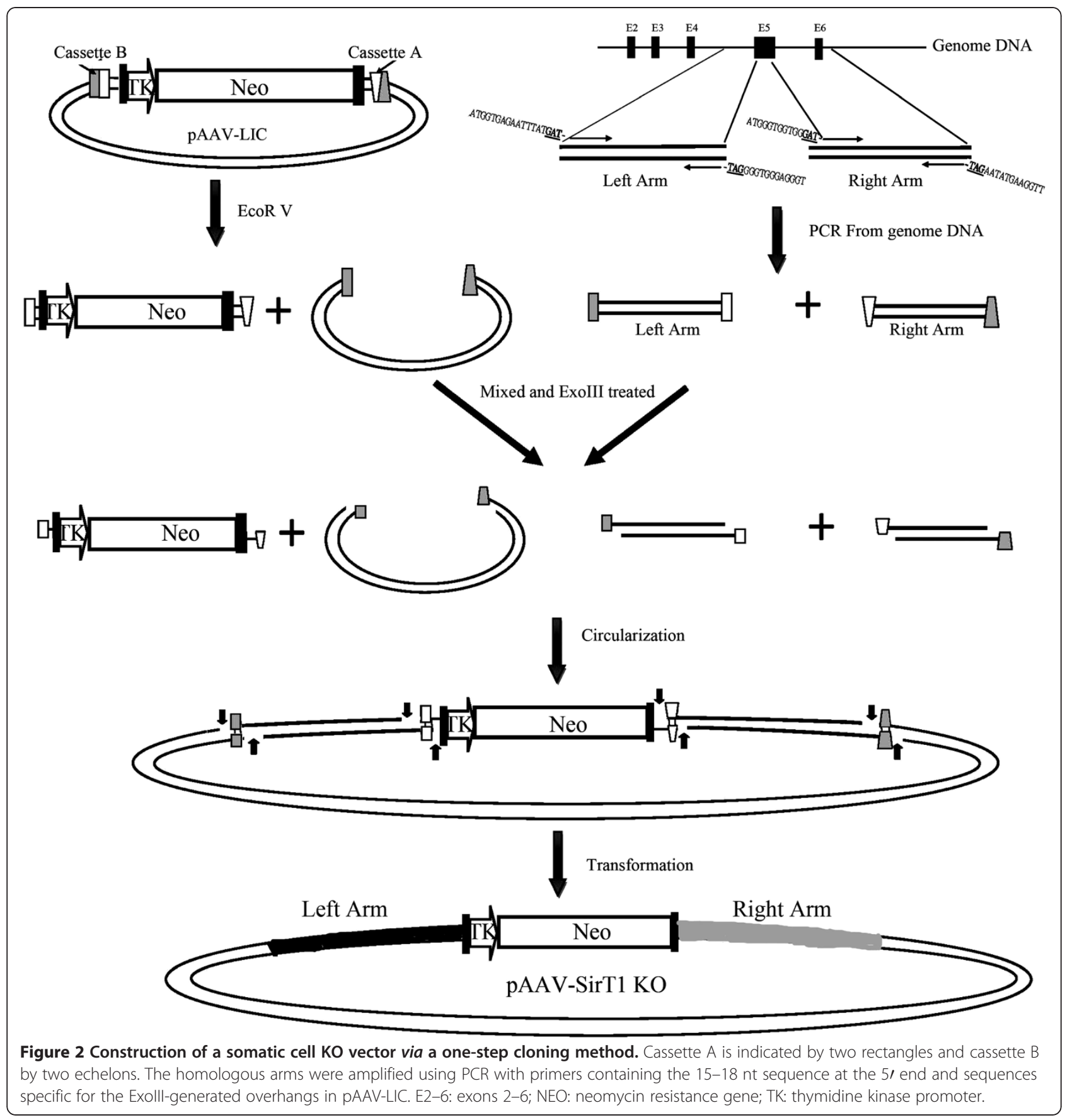

errors can be produced during amplification by PCR and it need high efficiency competent cells for the transformation.

\section{Methods}

Materials and bacterial strains

Restriction enzymes, $P f u$ polymerase, ExoIII and DNA ligation kits were purchased from Takara (Dalian, China). Kits for plasmid minipreps, DNA purification and genome extraction were purchased from Tiangen
(Beijing, China). Primers were purchased from Genscript (Nanjing, China). The E. coli DH5 $\alpha$ strain was used for plasmid preparation and all restriction-based cloning.

\section{Cell culture}

HEK-293T cells were cultured in Dulbecco's modified Eagle's medium (DMEM) containing 10\% fetal bovine serum (FBS) and 100 units/ml penicillin-streptomycin. The colorectal cancer cell line, HCT116, was cultured in McCoy's 5A containing 10\% FBS and 100 units $/ \mathrm{ml}$ 


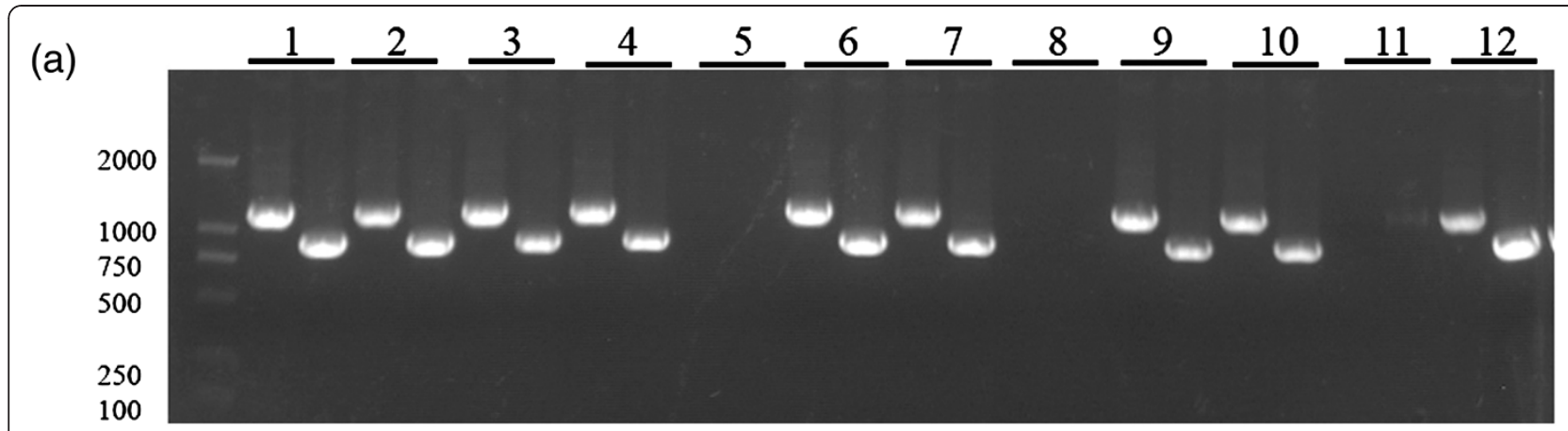

(b)

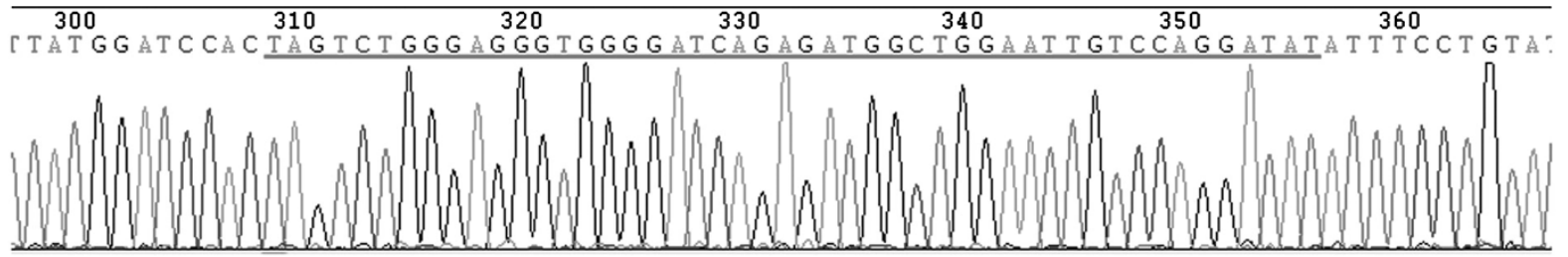

(c)

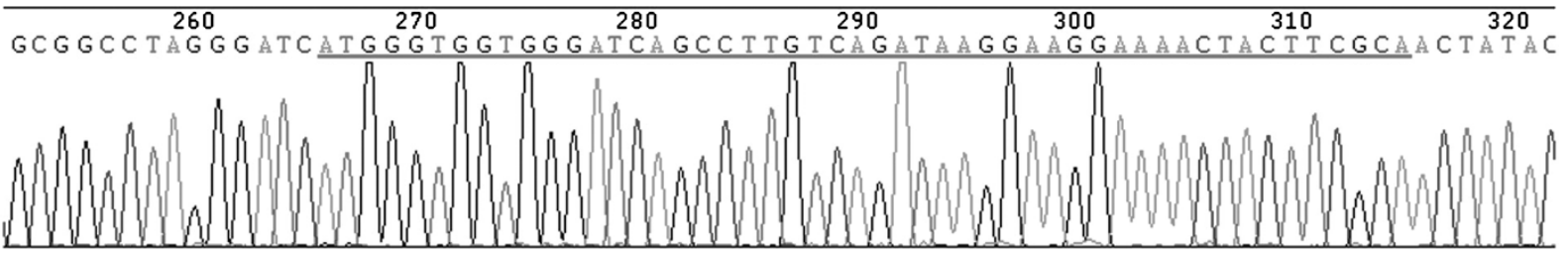

Figure 3 Colony PCR and sequencing results for the pAAV-SirT1 gene KO plasmid. (a) Colony screening by PCR. Left arm (> 1 kb) and right arm $(<1 \mathrm{~kb})$ positives are shown. (b) The sequence in the joint formed by the vector and the left arm of SirT1. The joint sequence is underlined in gray. (c) The sequence in the joint formed by the vector and the right arm of SirT1. The joint sequence is underlined in gray.

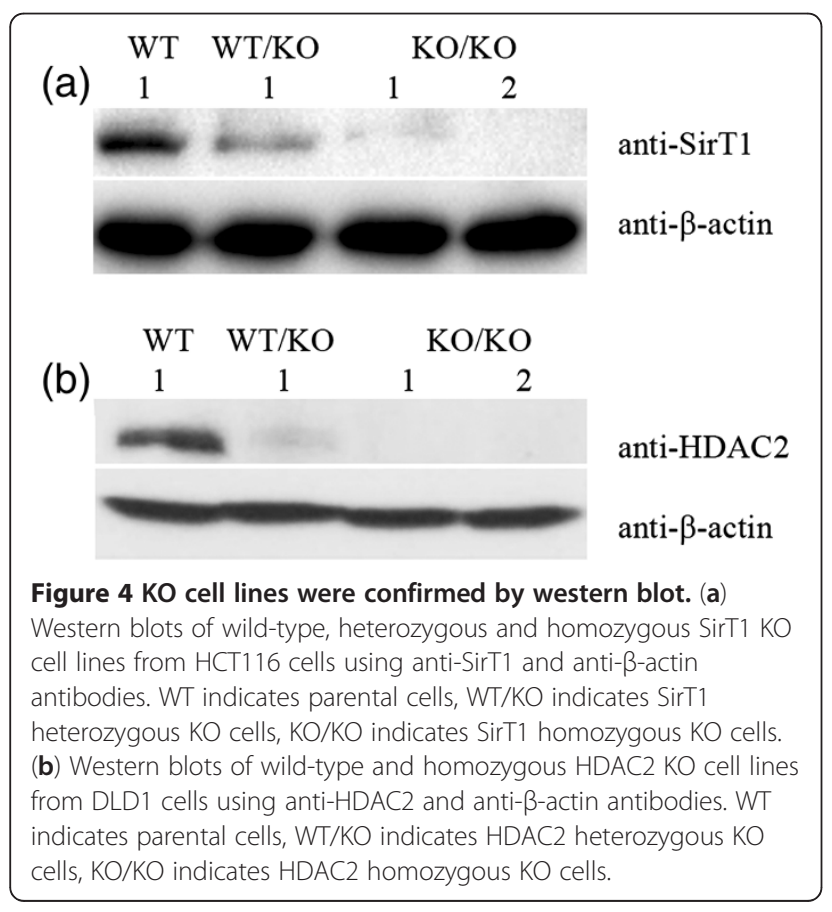

penicillin-streptomycin. McCoy's 5A medium was purchased from AppliChem (Seajet Scientific Co., Ltd., Beijing, CHINA). All other cell culture reagents were obtained from Life Technologies Corporation (Shanghai, CHINA).

\section{Construction of the modified AAV shuttle vector}

The AAV shuttle vector, pTK-LoxP-NEO-AAV, was modified to produce the pAAV-LIC vector. Two pairs of unique primers were designed for creating 'LIC zones'. These primer pairs were cassette A (sense, $5^{\prime}$-CAT GGG TGG TGG GAT ATC TTA TAC TTC CAA G-3'; and antisense, $5^{\prime}$-TCG ACT TGG AAG TAT AAG ATA TCC CAC CAC CCA TG-3') and cassette B (sense, $5^{\prime}$-CTA GCA TGG TGA GAA TTT ATG ATA TCC CCA CCC TCC CAG A-3'; and antisense, $5^{\prime}$-CTA GTC TGG GAG GGT GGG GAT ATC ATA AAT TCT CAC CAT G-3'). The underlined sequence indicates the recognition site for EcoRV.

Primers were diluted to $5 \mathrm{mM}$ with double-distilled water. Sense and antisense primers ( $3 \mu \mathrm{l}$ of each) for cassette A or cassette B were mixed with $3 \mu \mathrm{l}$ of buffer 3 
(NEB) and $21 \mu \mathrm{l}$ of sterile water. The reaction was incubated at $94^{\circ} \mathrm{C}$ for $10 \mathrm{~min}$, then cooled slowly to room temperature. Cassette A or B fusion products were inserted into the digested pTK-LoxP-NEO-AAV vector using DNA ligation kits. Recombinants were verified by restriction analysis and sequencing.

\section{General primers for AAV gene-targeting vectors}

The homologous arm is around $1 \mathrm{~kb}$, and we designed primers using primer3 (http://frodo.wi.mit.edu/primer3/). To insert arms into the pAAV-LIC backbone, the primers needed to contain complementary sequences to the ExoIII-generated overhangs in pAAV-LIC. The designed and synthesized primers were Left Arm Sense (5'-ATG GTG AGA ATT TAT GAT-3'), Left Arm Antisense ( $5^{\prime}$-TGG GAG GGT GGG GAT-3'), Right Arm Sense ( $5^{\prime}$-ATG GGT GGT GGG AT-3'), and Right Arm Antisense (5'-TTG GAA GTA TAA GAT-3').

\section{Construction of the SirT1 AAV KO vector}

Plasmid pAAV-LIC $(2 \mu \mathrm{g})$ was subjected to overnight digestion with $E c o R V$ in a $50 \mu \mathrm{l}$ reaction at $37^{\circ} \mathrm{C}$. The two fragments produced were purified using a gel extraction kit and diluted to $50 \mathrm{ng} / \mu \mathrm{l}$ in TE buffer (pH8.0). Two homologous arms were amplified from genomic DNA extracted from HCT116 cells. Two pairs of unique primers were designed: (a) SirT1, left arm, forward (5'ATGGTGAGAATTTATGATCGCCTGTTTGTATCCTTCCTGAC-3') and reverse (5'-TGGGAGGGTGGGGATCAGAGATGGCTGGAATTGTCC-3'); and (b) SirT1, right arm, forward (5'-ATGGGTGGTGGGATCAGCCTTGTCAGATAAGGAAG-3') and reverse $\left(5^{\prime}-\right.$ TTGGAAGTATAAGATCACCTTAGCTCGGCAGTTC TT-3'); (c) HDAC2, left arm, forward (5'- ATGGTGA GAATTTATGATAGCCAGCAGCATTGCTGCAG - $3^{\prime}$ ) and reverse (5'-TGGGAGGGTGGGGATAGCCGCG GAACCCAGCGCC $-3^{\prime}$ ); and (d) HDAC2, right arm, forward (5'-ATGGGTGGTGGGATAAGTCTGCTAC TACTACGAC $\left.-3^{\prime}\right)$ and reverse (5'-TTGGAAGTATAA GATCCCCAAAGCAGGGTCTAT $-3^{\prime}$ ). The underlined sequences indicate the designed primers from the SirT1 genome DNA or HDAC2 genome DNA. The PCRs were performed using $p f u$ polymerase, with amplicons electrophoresed and then recovered by gel extraction kits. Recovered fragments were diluted to $100 \mathrm{ng} / \mu \mathrm{l}$ in TE buffer.

The vector fragments and amplicons were mixed in one reaction tube. The large fragment $(1 \mu \mathrm{l})$, small fragment $(2 \mu \mathrm{l})$, left arm $(2 \mu \mathrm{l})$ and right arm $(2 \mu \mathrm{l})$ were mixed with $1 \mu \mathrm{l}$ of $10 \times$ ExoIII buffer and $1 \mu \mathrm{l}$ of sterile water. The tube was pre-chilled on ice for $2 \mathrm{~min}$, and then $1 \mu \mathrm{l}$ of ExoIII (20 units) was added to the tube and gently mixed. After a $1 \mathrm{~h}$ incubation at $0^{\circ} \mathrm{C}, 1 \mu \mathrm{l}$ of 0.5 $\mathrm{M}$ EDTA was added to stop the reaction. The enzyme was inactivated at $65^{\circ} \mathrm{C}$ for $5 \mathrm{~min}$. After cooling, the mixture was transformed into E. coli $\mathrm{DH} 5 \alpha$ and cultured at $37^{\circ} \mathrm{C}$ overnight. Positive transformants were identified by colony PCR and sequencing.

\section{Somatic cell gene targeting}

Somatic cell gene targeting was conducted as described previously [5]. Briefly, the targeting AAVs were packaged in 293T cells by transfecting equal amounts of SirT1 or HDAC2 KO targeting vector, pHelper and $\mathrm{pRC}$ plasmids (1 mg each). After $72 \mathrm{~h}$, scraped the transfected cells and suspended the cells into sterile phosphate-buffered saline, then freezed and thawed the pellet three cycles, finally spined the lysate to remove cell debris and divided the supernatant containing rAAV into several aliquots and frozen at $-80^{\circ} \mathrm{C}$. HCT116 or DLD1 cells were infected with the SirT1 or HDAC2 targeting viruses and selected with geneticin for two weeks. The geneticin-resistant clones were then screened for homologous recombination by genomic PCR with primers derived from the neomycin resistance gene (5'-GTTG TGCCCAGTCATAGCCG-3') and the upstream region of the left homologous arm (SirT1: 5'-GAAAGTTCCC CACATCTGCT-3'; HDAC2: 5'-TGCTCCAATCTTC CAGTGTCT- $3^{\prime}$ ). Positive clones were confirmed by genomic PCR, with primers derived from the neomycin resistance gene (5'-TCTGGATTCATCGACTGTGG-3') and the downstream region of the right homologous arm (SirT1: 5'-TCACTCCTCCAGGGCTAAAA-3'; HDAC2 5'-CACCTAGGAACAGCCTTTGC-3'). Correctly targeted clones were infected with adenoviruses expressing Cre-recombinase to delete the selectable drug marker. To select clones with successful deletion of the selectable drug marker, genomic PCR was employed to amplify an approximate $250 \mathrm{bp}$ fragment in which the LoxP site was inserted, using specific primers (SirT1:5'TAGGTGTGTGTCGCATCCAT- $3^{\prime}$ and $5^{\prime}$-CCTGTTC CAGCGTGTCTATGT-3'; HDAC2:5' - CATGGCGTACA GTCAAGGAG- $3^{\prime}$ and $5^{\prime}$-CAAATGTCGGTCCCTCCTC-3'). The heterozygous $\mathrm{KO}$ clones were infected with the same targeting virus to target the second allele and the neomycin resistance gene was excised as described earlier. Final confirmation in the generation of the KO cell lines was done using western blotting.

\section{Competing interests}

The authors declare they have no competing interests.

\section{Authors' contributions}

$\mathrm{RD}$ conceived the project and designed the experiments along with $\mathrm{YL}$. YL, $\mathrm{HZ}$ and SL performed the laboratory work and analyzed the results. $X Z$ and $R$ D wrote the manuscript. All authors read and approved the final manuscript.

\section{Acknowledgements}

This work was supported by grants from the National Basic Research Program of China (2011CB944404), the National Natural Science Foundation of China (30971499), the Trans-Century Training Programme Foundation for 
the Talents by the State Education Commission (NCET-10-0655), and the Fundamental Research Funds for the Central Universities (204275771).

Received: 21 June 2012 Accepted: 2 October 2012

Published: 9 October 2012

\section{References}

1. Sedivy JM, Dutriaux A: Gene targeting and somatic cell genetics-a rebirth or a coming of age? Trends Genet 1999, 15(3):88-90.

2. Hirata R, Chamberlain J, Dong R, Russell DW: Targeted transgene insertion into human chromosomes by adeno-associated virus vectors. Nat Biotechnol 2002, 20(7):735-738.

3. Porteus MH, Cathomen T, Weitzman MD, Baltimore D: Efficient gene targeting mediated by adeno-associated virus and DNA double-strand breaks. Mol Cell Biol 2003, 23(10):3558-3565.

4. Zhang X, Guo C, Chen Y, Shulha HP, Schnetz MP, LaFramboise T, Bartels CF, Markowitz S, Weng Z, Scacheri PC, et al: Epitope tagging of endogenous proteins for genome-wide ChIP-chip studies. Nat Methods 2008, 5(2):163-165.

5. Zhang P, Zhao Y, Zhu X, Sedwick D, Zhang X, Wang Z: Cross-talk between phospho-STAT3 and PLCgamma1 plays a critical role in colorectal tumorigenesis. Mol Cancer Res 2011, 9(10):1418-1428.

6. Rago C, Vogelstein B, Bunz F: Genetic knockouts and knockins in human somatic cells. Nat Protocols 2007, 2(11):2734-2746.

7. Liu P, Jenkins NA, Copeland NG: A highly efficient recombineering-based method for generating conditional knockout mutations. Genome Res 2003, 13(3):476-484

8. Angrand PO, Daigle N, van der Hoeven F, Scholer HR, Stewart AF: Simplified generation of targeting constructs using ET recombination. Nucleic Acids Res 1999, 27(17):e16.

9. Zhang Y, Buchholz F, Muyrers JP, Stewart AF: A new logic for DNA engineering using recombination in Escherichia coli. Nat Genet 1998, 20(2):123-128.

10. Li C, Wen A, Shen B, Lu J, Huang Y, Chang Y: FastCloning: a highly simplified, purification-free, sequence- and ligation-independent PCR cloning method. BMC Biotechnol 2011, 11:92.

11. Li MZ, Elledge SJ: SLIC: a method for sequence- and ligation-independent cloning. Methods Mol Biol 2012, 852:51-59.

12. Geu-Flores F, Nour-Eldin HH, Nielsen MT, Halkier BA: USER fusion: a rapid and efficient method for simultaneous fusion and cloning of multiple PCR products. Nucleic Acids Res 2007, 35(7):e55.

13. Du R, Li S, Zhang X: A modified plasmid vector pCMV-3Tag-LIC for rapid, reliable, ligation-independent cloning of polymerase chain reaction products. Anal Biochem 2011, 408(2):357-359.

14. Gibson DG, Young L, Chuang RY, Venter JC, Hutchison CA 3rd: Smith HO: Enzymatic assembly of DNA molecules up to several hundred kilobases. Nat Methods 2009, 6(5):343-345.

15. Gibson DG, Smith HO, Hutchison CA 3rd, Venter JC, Merryman C: Chemical synthesis of the mouse mitochondrial genome. Nat Methods 2010, 7(11):901-903.

16. Gibson DG: Synthesis of DNA fragments in yeast by one-step assembly of overlapping oligonucleotides. Nucleic Acids Res 2009, 37(20):6984-6990.

17. Hsiao K: Exonuclease III induced ligase-free directional subcloning of PCR products. Nucleic Acids Res 1993, 21(23):5528-5529.

\section{Submit your next manuscript to BioMed Central and take full advantage of:}

- Convenient online submission

- Thorough peer review

- No space constraints or color figure charges

- Immediate publication on acceptance

- Inclusion in PubMed, CAS, Scopus and Google Scholar

- Research which is freely available for redistribution 